\title{
The Post Hoc Procedure in Survival Analysis for Undergraduate Students Performance
}

\author{
Azme Khamis ${ }^{1 *}$, Che Azmeeza Che Hamat ${ }^{2}$ and Mohd Asrul Affendi Abdullah ${ }^{1}$ \\ ${ }^{1}$ Department of Mathematics and Statistics, Faculty of Applied Sciences and Technology, Universiti Tun \\ Hussein Onn Malaysia, 84600 Pagoh, Johor, Malaysia. \\ ${ }^{2}$ Faculty of Applied Sciences and Technology, Universiti Tun Hussein Onn Malaysia, 84600 Pagoh, Johor, \\ Malaysia.
}

Received 30 September 2017; accepted 3 June 2018; available online 1 August 2018

DOI: https://10.30880/jst.2018.10.02.024

\begin{abstract}
Survival analysis is a term used to describe the analysis of data in the form of times from a welldefined time origin until the occurrence of any specific event. In an academic research, the time origin often corresponds to the recruitment of an individual into an experimental study. There are the unmeasured chance of finding a falsely significant difference between two or more groups. Compared more than two groups simultaneously increased the chance of making type 1 error. This paper proposed survival analysis with multiple comparison studies to came up with this issue which is to identify the best undergraduate student performance based on the three certificates of qualification which Diploma, Matriculation and STPM. The undergraduate student achievement data are taken to explain this methodology. Kaplan-Meier plotted with survival comparison test, Log-rank test is used to elaborate the application of the Scheffe test. The result reveals that the undergraduates' students from STPM have performed better in Degree. The Kaplan-Meier curve shows a significant difference in survival plot among three certificates of qualification. However, $p$-value adjusted by Scheffe test for paired Matriculation and Diploma was found an insignificant difference. So, this study shows the importance of $p$-value adjustment with Scheffe test in comparing more than two groups to draw a right conclusion.
\end{abstract}

Keyword: Survival Analysis; Kaplan-Meier; Log-rank test; Scheffe Test; undergraduate students.

\section{Introduction}

The three entrance requirement of the Institution of Students who are given the chance to receive higher education in public university in Malaysia are subsidized by the government. The three entrance requirement of the Institution of High Education (IHE) available to pursue the Degree in Universiti Tun Hussein Onn Malaysia are Diploma, Matriculation and STPM. The majority of Matriculation students are Bumiputera and Chinese for STPM [1]. The selection criteria for Matriculation and STPM students are under the control of Ministry Institution of Higher Education (IHE). However, universities have an authority to select and determine the credential of students during Degree.

The performances of students are obtained by measuring the learning assessment and curriculum [2]. It is well accepted that the quality of the graduates is often measured by their class degrees or the final grades earned which is commensurate with Grade point Average (GPA) achieved [3].

Many of students actually entered with a low academic performance but improved while in university to the point of becoming an excellent student. Hence, the level of achievement of students before entering university does not determine the level of achievement of students while in university.

Currently, many techniques propose to evaluate the students' performance. Data mining is the common techniques that widely used in education area especially for analysing students' performances [4]. The process of study using the data mining extracted a useful information and pattern from a huge educational database [5]. This study, therefore, assisted the educator in providing an effective approach to study on students' performance.

Survival analysis is a statistical procedure that has time to event data and for data analysis in which the outcome of interest is time until an event occurs [6]. In contrast, survival analysis allows inclusions of 
information for those who were failed during the study [7].

Survival analysis that applied in an academic sector used to only determine whether the groups of the study had a significantly different survival time [8]. Rottenly, the researchers are interested in comparing more than two groups of treatments using the survival curves [9]. When this is done, the chance of making at least type 1 error or finding a falsely significant difference between any two groups is increased above the desired level.

By applying the survival analysis method, the chance to make at least type 1 error or finding a falsely significant difference is high. Problems are arises when more than two groups exist in selecting the best groups in the study. The log-rank test and Kaplan Meier estimator in the survival analysis conclude the results without any adjustment resulting in excessing of produce type 1 error. The applicable method to handle in comparison respective group of undergraduate students is decided by using multiple comparisons procedures or multiple hypothesis testing. The key goal of multiple hypothesis testing are to limit or at least measure of type 1 errors result [10].

Many techniques proposed by previous researchers for more than two groups of study. Post Hoc procedure is the hypothesis testing method in this multiple hypothesis testing studies. Recently, more than twenty tests in Post Hoc procedure are available. Several post hoc procedures for pairwise comparison like Dunnet [11], Tukey [12], Scheffe [13] and Duncan test [14] which use the Bayesian inference are used.

Every test has its own advantages and disadvantages. The previous researcher has used Bonferroni correction because of it is simple and easy to handle for a small data [10]. This study different with the previous researcher. The Scheffe test is generally applied to unequal sample size. Since this study involves the different number of sample size for each group which are Diploma, Matriculation and STPM by each semester, the Scheffe test is the most appropriate Post Hoc procedure to used and applied for this study compared to the Bonferroni correction.

The use of Scheffe test in survival analysis for students is randomly seen. The GPA students from Diploma, Matriculation and STPM are compared by using the Scheffe test. This is the main motivation behind this research attempt to explore the application of multiple hypothesis testing in survival analysis. The Kaplan-Meier plot and the logrank test are used to compare the survival of students in a different group (Diploma, Matriculation and STPM).

In this paper, survival analysis with multiple hypothesis testing is performed on undergraduate students in UTHM. This paper proposed survival analysis with multiple hypothesis testing to come up with this issue which is to determine which undergraduate student perform better during the Degree is based on the GPA obtains by each semester.

The next section of this paper focused on the methodology used in comparing and estimated students' performance using multiple comparison procedures and survival analysis. Then, the result and discussion is described in section 3. Finally, the conclusion is outlined in Section 4.

\section{Methodology}

This research focuses on the undergraduate students' in UTHM for intake $2011 / 2012$. The data is collected from the Students' Academic Department (PPA). Hence, this study focused on the GPA obtained for every semester from Diploma, Matriculation and STPM students. The statistical packages used for this research are the SPSS and R package. The SPSS software used for multiple hypothesis testing used Scheffe test in Post Hoc procedure and $\mathrm{R}$ package for the analysis of survival of undergraduate students using the Kaplan Meier method and Log rank test. KaplanMeier, log-rank test and Post Hoc adjustment method (Scheffe test) are practiced to fulfill the result of survival analysis with the Post hoc comparison.

The independent variable for undergraduates certificates of qualification before pursues the Degree are taken in the studies to find out their effect on student performance in UTHM. The variable is divided into three certificates of qualifications has the first category as Diploma, the second category defined Matriculation and the third category covers the STPM students who pursues the Degree in UTHM. 


\subsection{Kaplan-Meier estimation of the survival function}

The Kaplan-Meier (product-limit) method was a special case of the life table technique in which the series of time intervals were formed in such a way that only one event occurred in each time interval [15]. Suppose that there are $n$ individuals with observed survival times $t_{1}, t_{2}, t_{3} \ldots, t_{n}$ and $r$ is the failure time among the individuals where $r<n$. The ordered failure times

$$
\begin{gathered}
t_{(j)}, j=1,2, \ldots, r= \\
t_{(1)}<t_{(2)}<\ldots<t_{(r)} .
\end{gathered}
$$

Let $n_{j}$ which $j=1,2, \ldots, r$ be the number of individuals who were success just before the time $t_{(j)}$ and let $d_{(j)}$ be the number of individuals failed at time $t_{(j)}$. The probability for an individual failed during the interval $t_{(j-1)}$ to $t_{(j)}$ is estimated by

$$
\frac{d_{(j)}}{n_{(j)}}
$$

Therefore the corresponding estimated survival probability in that interval is

$$
\frac{\left[n_{(j)}-d_{(j)}\right]}{n_{(j)}}
$$

If the censored survival times and one or more failure times were same, then, in this case, it is assumed that the censored survival time is taken to occur immediately after the failure time. So, the estimated survival function for any time in the $t$ in the $j^{t h}$ constructed time interval from $t_{(j)}$ to $t_{(j+1)}, j=1,2, \ldots, r \quad$ and all the preceding time intervals is led to the following Kaplan-Meier estimate of the survivor function,

$$
\hat{S}(t)=\prod_{t \leq t_{(j)}} \frac{n_{j}-d_{j}}{n_{j}}
$$

For $t_{(j)} \leq t<t_{(j+1)}, j=1,2, \ldots, r, \hat{S}(t)=1$ for $t<t_{(1)}, \hat{S}(t)=0$ for $t \geq t_{(r)}$ if $t_{(r)}$ is the last observation.

\subsection{Statistical test using Log-rank test}

The log-rank test is the nonparametric statistical test used for the comparison of two or more groups of survival data. These tests are used for the comparison of three groups in the undergraduate student's survival data. The hypothesis testing for this log-rank test as:

$H_{0}$ : All survival curves were the same

$H_{1}$ : All survival curves were difference

Log-rank statistics for more than two groups involves variances and covariance of observed values minus expected values, $\left(O_{i}-E_{i}\right)$. The log rank statistics $\sim X_{1}^{2}$ with $G$ -1 degree of freedom, $d f$.

The variation of the log-rank test derived by applying different weights at the $j^{\text {th }}$ failure time. Weighting the Test statistics:

$$
\frac{\left(\sum_{j} w\left(t_{j}\right)\left(m_{i j}-e_{i j}\right)\right)^{2}}{\operatorname{Var}\left(\sum_{j} w\left(t_{j}\right)\left(m_{i j}-e_{i j}\right)\right)}
$$

$\sum_{j} w\left(t_{j}\right)=$ Weight at $j^{\text {th }}$ failure time

Number of failures $=m_{i j}$

Expected cell counts $=e_{i j}$

This can be seen by relative weight assigned to the test. The log rank test was optimal under proportional hazard assumptions. The Breslow test had high power under the proportional hazard assumptions.

\subsection{Post Hoc procedures using the Scheffe test method}

ANOVA analysis is used to examine the significant differences among the mean GPAs obtained from Semester 1 until Semester 8 of the students for two and more groups which Diploma, Matriculation and STPM. After the analysis, if the $F_{\text {value }}$ is largest and $H_{0}$ rejected, the Scheffe test will conduct to identify which qualification performs better during the Degree.

The decision of the One-Way Analysis of Variance is to reject the null hypothesis, meaning that at least one of the means is not the same as the other means. The mean comparison is conducted after the one-way ANOVA to figure out which variables actually difference. The mean comparison using Scheffe test is used in this analysis. This test 
analyse the pairs of means to see if the differences are exist.

This test is set up to test if pairs of means are different. The formulas refer to mean sample $i$ and mean sample $j$. The values of $i$ and $j$ are varied, and the total number of tests will be equal to a combination of $k$, the number of samples.

$$
\begin{aligned}
& H_{0}: \mu_{i}=\mu_{j} \\
& H_{1}: \mu_{i} \neq \mu_{j}
\end{aligned}
$$

The Scheffe test is customarily used with unequal sample sizes, $N$. The critical value for the Scheffe test is the degrees of freedom for the between variance times the critical value, $\mathrm{CV}$ for the one-way ANOVA. This simplifies to be:

$$
\mathrm{CV}=(k-1) F(k-1, N-k, \alpha)
$$

Test statistics for the Scheffe test, $F_{S}$

$$
F_{s}=\frac{\left(\bar{x}_{i}-\bar{x}_{j}\right)^{2}}{S_{w}^{2}\left(\frac{1}{n_{i}}+\frac{1}{n_{j}}\right)}
$$

Number of sample group $i: n_{i}$

Number of sample group $j: n_{j}$

Sample mean of group $i: \bar{x}_{i}$

Sample mean of group $j: \bar{x}_{j}$

Sample variance: $S_{w}^{2}$

The significance level of students pursues the Degree is determined. When the number of comparisons became large, the test became too conservative and no longer allowed to find any variables was significant [16]. So for an overall comparison, if $p$-value less than 0.05 then considered difference is statistically significant. And pairwise comparison is significant if the $p$-value less than $5 \%$ divided by three groups $=1.7 \%$. So for an overall comparison if the $p$-value is less than 0.05 then considered difference would be statistically significant. And pairwise would be statistically significant if the $p$-value is less than $1.7 \%$.

\section{Result and Discussion}

The Diploma (D), Matriculation (M) and STPM (S) students in UTHM is analysed based on the semesters enrolled using the multiple comparison procedures and KaplanMeier. One of the best featured of KaplanMeier estimator is the estimating of survival curve, plotting can summarize the patterns of response and survival curves can visually compare [17].

In this study, Kaplan-Meier curves are used to describe the survival times of groups of students based on the qualifications. The Kaplan-Meier result summarizes survival data in terms of the number of events and the proportion surviving at each event time point. The time of this study is the number of semesters enrolled by Undergraduate students. The event from this study is student's achievement-GPA > 2.0 during Degree based on a number of the semester enrolled by students during the Degree. From this result, the proportion of survival students slightly decreased when students enrolled the study.

The Kaplan Meier survival estimate outlines the survival data in terms of the number of events and the proportion of surviving at each time to event point. Time in this study is the semesters enrolled by the students. The Kaplan Meier estimates can help explain the curve and the way the curve ends. The result of Kaplan Meier estimate for overall students can clearly see in Table 1.

In Table 1, three students have an event of GPA > 2.00 for all students pursue the Degree only 1 semester, 34 for enrolled 2 semesters, 17 for 3 semesters, 48 for 4 semesters and 107 for students enrolled 5 semesters. The number of events increased for students enrolled 6 semesters since many of the students performed well (GPA > 2.00) during the Degree. Since many Diploma students enrolled only 6 semesters, the number of events (students survives) decreased for students enrolled 7 semesters and increased for 8 semesters. The survival probability plot using Kaplan-Meier estimation can be seen in detail in Fig. 1. 
Table 1 Kaplan Meier result for eight semesters for overall students achievement data

\begin{tabular}{cccc}
\hline Semester enrolled & No. of risk & No of event & Survival probability \\
\hline 1 & 17562 & 3 & 0.9998 \\
2 & 17511 & 34 & 0.9979 \\
3 & 17441 & 17 & 0.9969 \\
4 & 17398 & 48 & 0.9942 \\
5 & 17327 & 107 & 0.9880 \\
6 & 17200 & 4511 & 0.7289 \\
7 & 12650 & 2888 & 0.5625 \\
8 & 9615 & 9118 & 0.0291 \\
\hline
\end{tabular}

The event from this study is Bachelor's Degree student result with GPA > 2.0 during the semester of study from Semester 1 until Semester 8. From Table 1, the proportion of survival Bachelor's Degree students slightly decreased when students enrolled more than six semesters.

Kaplan-Meier plot is displayed in Fig. 1 to show the survival plot of students along time (semester enrolled) among various groups which are Diploma, Matriculation and STPM. Survival experienced or students' performance of Diploma are completely different from Matriculation and STPM groups of Bachelor's Degree students based on the certificates of qualification. In the early stage of the study, every category has approximately same survival of students' performance but with time being, the width of a curve in Fig. 1 became wider. Fig. 2 is plotted for a clear explanation. This Fig. 2 showed that a big difference survival probability of STPM students compared to Diploma and Matriculation.

To find out that whether these differences occurred by chance or the difference is really significant, the log-rank test is performed with the post hoc comparison for each pair of a group in every variable. The post hoc adjusted $p$-value was calculated by Scheffe test. Both variables shows the overall significant differences among groups. Meanwhile, in order to find out which pairs group is a significantly different, the test is done by using Scheffe test. The log-rank test is used to compare the survival times of Diploma, Matriculation and STPM. The null hypothesis for a log-rank test is the group that has the same survival. The log-rank test has a chisquared distribution, $\chi^{2}$ with two degrees of freedom. The $\chi_{\text {value }}^{2}=13542$ from the analysis is higher than $\chi^{2}{ }_{\alpha=0.05}=5.991$. It indicates that the certificates of qualification of Degree students are statistically significant in survival. Hence, it shows that these certificates of qualifications has a difference survival time when pursues the Degree in UTHM at the significance level of 0.05 . It can be concluded that there are significant difference between STPM curve, Diploma curve and Matriculation curve by using the log-rank test.

Table 2 shows the variables of students' level of qualifications in pairwise (Post Hoc) comparison, the pair Matriculation and STPM, $(\mathrm{M}, \mathrm{S})$ is significant in the case of adjusted $p$ value by Scheffe test for all of the semester test. The other pair Diploma and STPM (D, S) presented out as a statistically significant difference in the survival pattern by all the semesters except in Semester 6. The other pair Diploma and Matriculation, (D, M) highlighted as a statistically significant difference in the survival pattern in Semester 1, Semester 2, Semester 3, Semester 6 and Semester 8. The other semester for the pair (D, M) found as significant in the case of nonadjusted $p$-value using log-rank test with a $p$ value less than 0.0001. But these pairs (D, M) did not show the significant differences after the $p$-value adjusted by Scheffe test. 


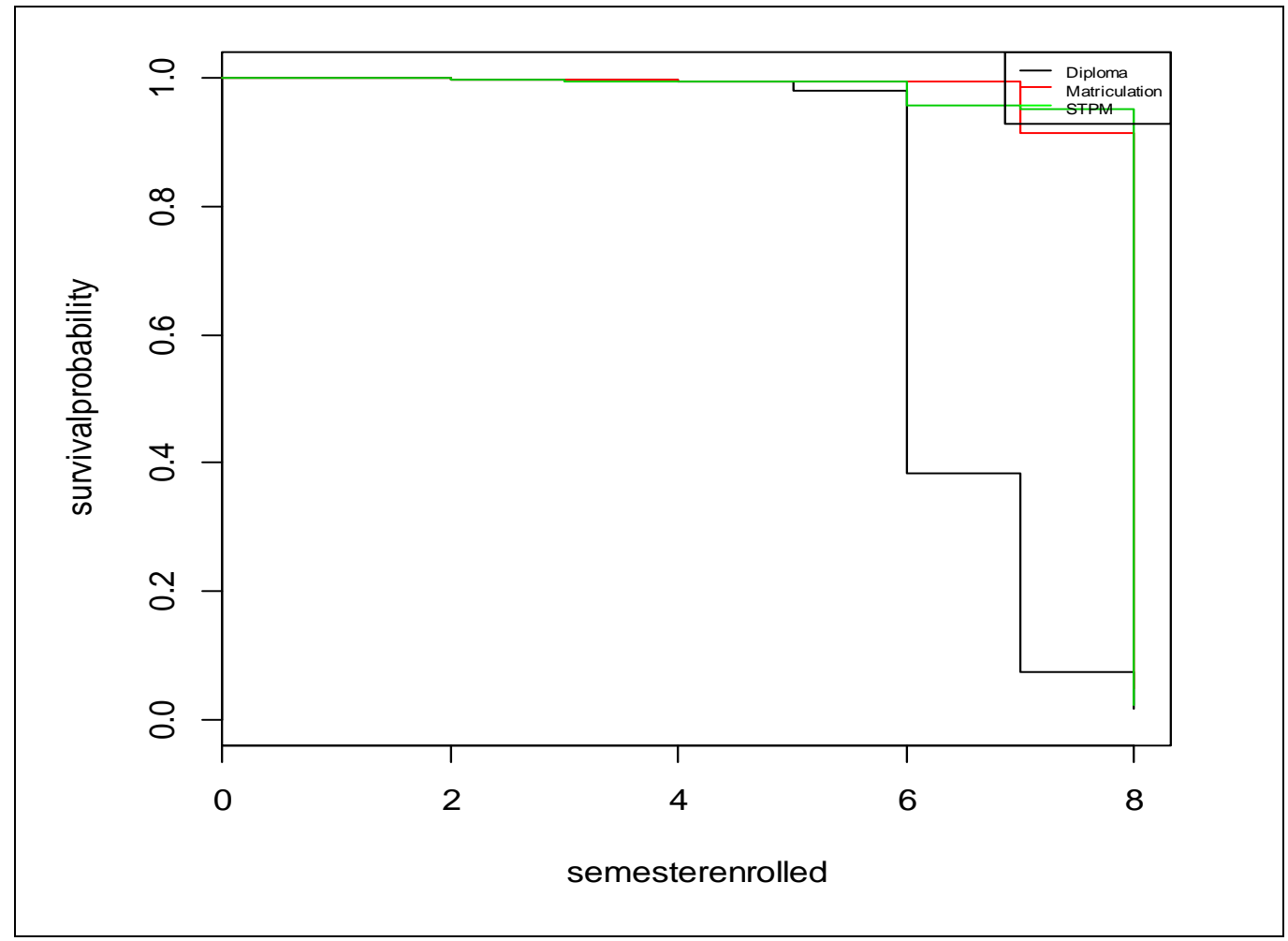

Fig. 1 Survival probability plot using Kaplan Meier estimation for each qualification

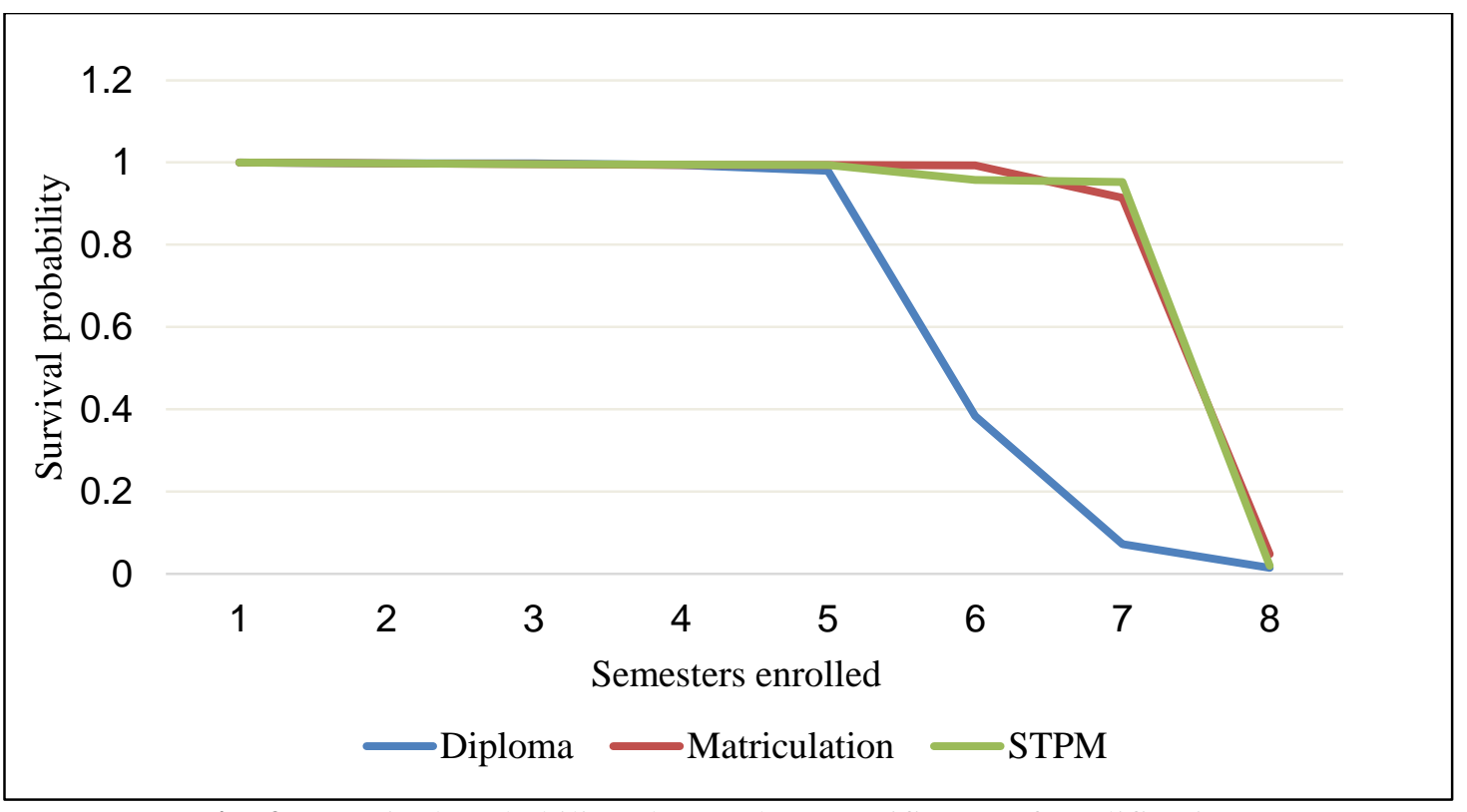

Fig. 2 Survival probability plot on three certificates of qualifications. 
Table 2 Comparison of survival pattern for selected variable.

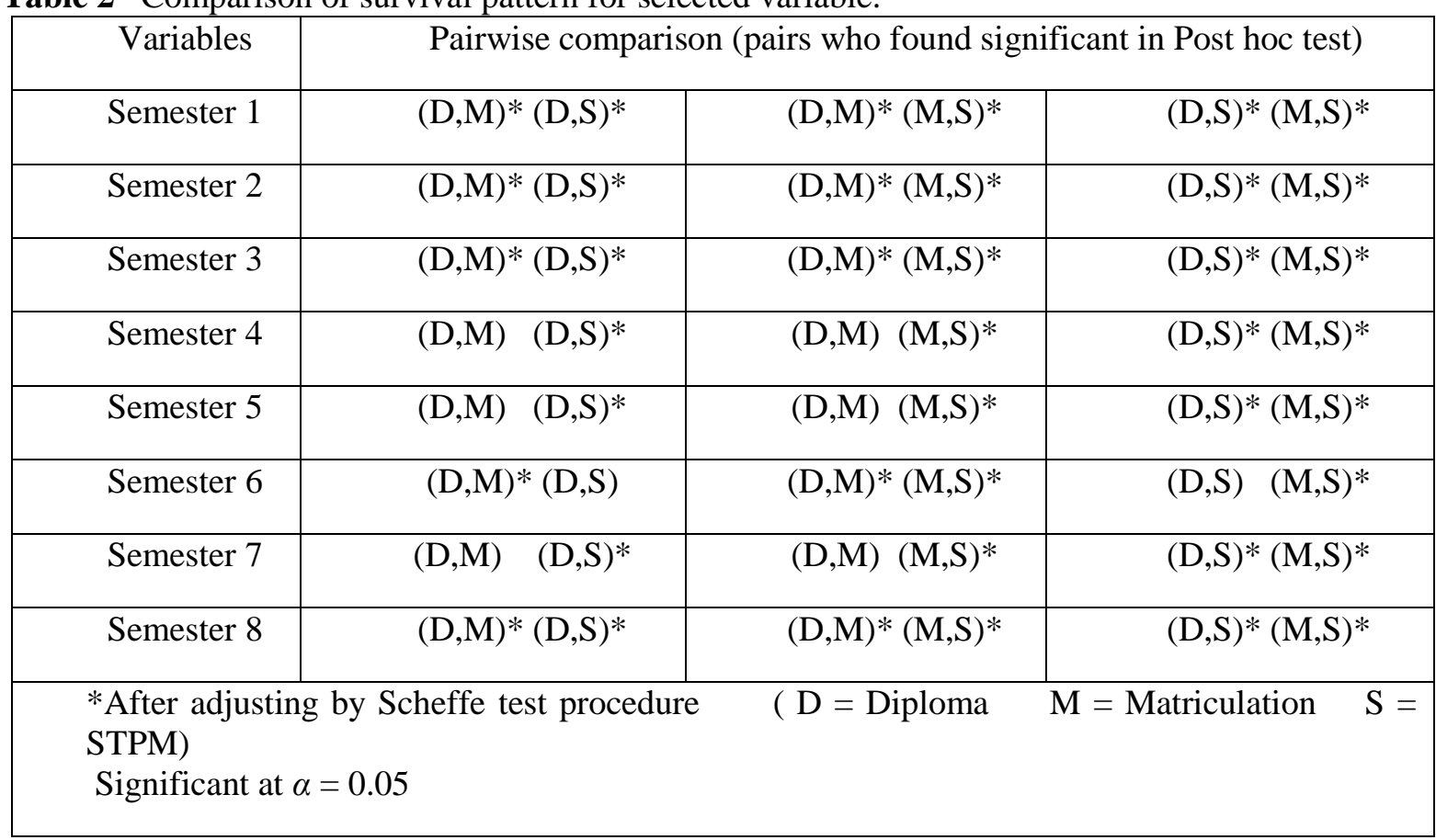

\subsection{Discussion}

The multiple hypothesis testing with adjust of $p$-value is concerned by the statisticians since a long time $[18,19]$. The $p$-value adjustments fixed in the ANOVA study and in common hypothesis procedure. There are frequently no exact adjustment method obtains for comparisons more than two groups to calculate adjusted $p$-value directly in survival analysis.

The comparison of three groups using the Log-rank test in survival analysis methods for three groups are not available to handle since it concludes with all the general. The Log-rank test only stated that there are significant difference in students' performance by Diploma, Matriculation and STPM when pursuing the Degree at $\alpha=0.05$.

Accordingly, categorize the two groups for each independent variable for multiple comparisons using Scheffe test for analysis using a pair (D, M) and a pair (D, S).

By applying of Scheffe test, groups of students are significant difference can determined. The data is taken from student achievement data for the study on student survival and the independent variables Semester 1 until Semester 8 are interpreted as simple analysis when using Scheffe test.
All the analysis yields the Kaplan-Meier curve reveals there are significant difference in survival among independent variables which is semesters for three certificates of qualification using a Log-rank test. After the analysis was further for Post-hoc or multiple comparisons, the Kaplan-Meier curve shows a clear difference in category Diploma, STPM and Matriculation, STPM.

The Post Hoc and Kaplan-Meier curve show a statistically significant difference in the category of Diploma (D), Matriculation (M) and STPM (S).

The study reveals the significant difference among Bachelor's Degree student survival among Diploma, Matriculation and STPM. The Scheffe test finding is supported by a Kaplan-Meier curve. But in a case of Post-Hoc comparison using Scheffe test, category Diploma and STPM (D, S) and Matriculation and STPM (M, S) show a clear difference in survival pattern of students.

From the Log-rank test for non-adjusted $p$-value, the Kaplan-Meier curve is also illustrates the difference survival for each of the qualifications but a vaguely not far from the pattern in starting of the survival curve in Figure 1. After the $p$-value adjusted by the Scheffe test for comparison a pair Diploma, Matriculation and it founds an insignificant 
difference between students from Diploma and Matriculation for Degree students' survival.

Thus, it can be summarized that the survival analysis that applies Post Hoc comparison and survival analysis methods show that the STPM has a significant difference when compared with students from Diploma and Matriculation. So, the pair of study for Diploma, Matriculation and STPM gives a correction of $p$-value using Scheffe test and reveals the importance of $p$-value adjustment in multiple hypothesis testing to sum up it all.

\section{Conclusion}

The students' performance shows that 95\% confident that the Diploma, Matriculation and STPM are significantly different. In this research, it noted that the multiple comparison procedures and Kaplan-Meier estimator analysed well to the analysis of students' performance for student intake 2011/2012 in University Tun Hussein Onn Malaysia.

Since the survival analysis cannot determine which pair was significant, hence, the Post Hoc that adjust the $p$-value is calculated by Scheffe test. Moreover, these methods are proposed because it is suits to use for not equal sample size used for each category for the study and a large number of samples.

The use of nonparametric survival models greatly reduce a cost, a number of sample size and time to follow up. From this study, it concludes that the survival curve of students from Diploma, Matriculation and STPM are different. All of the methods that has applied in this study agrees that the STPM students in UTHM performed better in Degree for intake 2011/2012.

Additionally, the accuracy of the results has been high since the methods proposed produced the consistent result. These methods well estimated the survival curve of different qualifications based on the semester enrolled by the students. The research using nonparametric survival model gave many advantages. Besides that, the specific qualifications that gave a good performance during the study are also determined. However, the Kaplan-Meier estimator is low compared to the efficiency obtained under the parametric setup [20].

\section{Acknowledgements}

The authors would like to thank University Tun Hussein Onn Malaysia, Academic Students' Department (PPA) for providing us with the secondary data of the student achievement and valuable source of information. We thank the editor, associate editor, and the reviewers for their constructive comments that improved the quality of this article. This research is sponsored, in part, by Postgraduate Research Grant (GPPS) U604.

\section{References}

[1] Hazilah, M. A., Aisyah, H., Hazura, M., Saidah, S. and Ibrahim, M. (2013). "Academic Performance of Pre-Graduate Students" in International Education Studies. Vol. 6. No. 6 pp. 131-136.

[2] Mat, U.N., Buniyamin, P.M., Arsad, R. and Kassim. (2013). "An Overview of Using Academic Analytics to Predict and Improve Students Achievement: A Proposed Proactive Intelligent Intervention" in Engineering Education (ICEED). IEEE $5^{\text {th }}$ Conference, pp.126130.

[3] Amirah, M.S., Wahidah, H. and Nuraini, A.R. (2015). "A Review on Predicting Students' Performance Using Data Mining Techniques". Elsevier. Vol. 72 pp. 414-422.

[4] Romero, C. and Ventura, S. (2010). "Educational Data Mining: A Review of the State of the Art, Trans" in Sys. Man Cyber Part C. Vol. 40. No. 6 pp. 601-618.

[5] Angeline, D.M.D. (2006). "Association Rule Generation for Student Performance Analysis Using Algorithm" in The SIJ Transactions on Computer Science Engineering \& its Applications (CSEA). Vol. 1. No. 1 pp. 12-16.

[6] Kleinbaum, D.G. (1996). "Survival Analysis: A Self-Learning Text". Springer-Verlag, New York.

[7] Youngkyoung, M., Guili, Z., Russeli, A.L., Timothy, J.A. and Matthew, W.O. (2011). "Nonparametric Survival Analysis of the Loss Rate of Undergraduate Engineering Students" in Journal of Engineering Educations. Vol. 100. No. 22 pp. 349-373.

[8] Tamada, Mike and Inman, C. (1997). "Survival Analysis of Faculty Retention 
Data: How Long Do They Stay" AIR national conference Orlando FL.

[9] Hosmer, D.W. and Lemeshow, S. (1999). Applied Survival Analysis. John Wiley and Sons, New York.

[10] Arunabh, T. and Anant, P. (2017). "Post Hoc Comparison in Survival Analysis: An easy approach" in Journal of Bioscience and Medicines, Vol. 5. pp. 112-119.

[11] Saville, D.J. (1990). "Multiple Comparison Procedures: The Practical Solution" in American Statistician, Vol. 44. pp. 174-180.

[12] Abdi, H. and Williams, L.J. (2010). Turkey's Honestly Significant Difference (HSD) Test. Encyclopedia of Research Design. Sage, Thousand Oaks.

[13] Scheffe, H. (1999). The Analysis of Variance. John Wiley \& Sons, Hoboken.

[14] Duncan, D.B. (1955). "Multiple Range and Multiple F Tests" in Biometrics, Vol. 11. pp. 142.

[15] Collett D. (1994). Modelling Survival Data in Medical Research, London: Chapman and Hall.

[16] Cox D.R. and Oakes D. (1984). Analysis of Survival Data. London. Chapman and Hall edition.

[17] Greenhouse, J., Stangl, D., and Bromberg, J. (1989). "An introduction to Survival Analysis: Statistics Method for Analysis of Clinical Trial Data" in Journal of Consulting Clinical Psychology, Vol. 57. No. 4 pp. 536-544.

[18] Scheffe, H. (1999). The Analysis of Variance. John Wiley \& Sons, Hoboken.

[19] Richter, S.J. and McCann, M.H. (2012). "Using the Tukey-Kramer Omnibus Test in the Hayter-Fisher Procedure" in British Journal of Mathematical and Statistical Psychology, Vol. 65. pp. 499-510.

[20] Greenhouse, J., Stangl, D., and Bromberg, J. (1989). "An introduction to Survival Analysis: Statistics Method for Analysis of Clinical Trial Data" in Journal of Consulting Clinical Psychology, Vol. 57. No. 4 pp. 536-544. 